SLAC-PUB-9797

hep-ex/9702007

CLNS 97/1461

CLEO 97-2

\title{
Study of the $B^{0}$ Semileptonic Decay Spectrum at the $\Upsilon(4 \mathrm{~S})$ Resonance
}

\begin{abstract}
We have made a first measurement of the lepton momentum spectrum in a sample of events enriched in neutral $B$ 's through a partial reconstruction of $B^{0} \rightarrow D^{*-} \ell^{+} \nu$. This spectrum, measured with $2.38 \mathrm{fb}^{-1}$ of data collected at the $\Upsilon(4 \mathrm{~S})$ resonance by the CLEO II detector, is compared directly to the inclusive lepton spectrum from all $\Upsilon(4 \mathrm{~S})$ events in the same data set. These two spectra are consistent with having the same shape above $1.5 \mathrm{GeV} / \mathrm{c}$. From the two spectra and two other CLEO measurements, we obtain the $B^{0}$ and $B^{+}$semileptonic branching fractions, $b_{0}$ and $b_{+}$, their ratio, and the production ratio $f_{+-} / f_{00}$ of $B^{+}$and $B^{0}$ pairs at the $\Upsilon(4 \mathrm{~S})$. We report $b_{+} / b_{0}=$ $0.950_{-0.080}^{+0.117} \pm 0.091, b_{0}=(10.78 \pm 0.60 \pm 0.69) \%$, and $b_{+}=(10.25 \pm 0.57 \pm 0.65) \%$. $b_{+} / b_{0}$ is equivalent to the ratio of charged to neutral $B$ lifetimes, $\tau_{+} / \tau_{0}$.
\end{abstract}

Submitted to Physical Letters B 
M. Artuso, ${ }^{1}$ A. Efimov, ${ }^{1}$ F. Frasconi, ${ }^{1}$ M. Gao, ${ }^{1}$ M. Goldberg, ${ }^{1}$ D. He,${ }^{1}$ S. Kopp,${ }^{1}$

G. C. Moneti, ${ }^{1}$ R. Mountain,${ }^{1}$ Y. Mukhin,${ }^{1}$ S. Schuh, ${ }^{1}$ T. Skwarnicki, ${ }^{1}$ S. Stone,${ }^{1}$

G. Viehhauser ${ }^{1}$ X. Xing, ${ }^{1}$ J. Bartelt,${ }^{2}$ S. E. Csorna,${ }^{2}$ V. Jain,${ }^{2}$ S. Marka,${ }^{2}$ A. Freyberger,${ }^{3}$

R. Godang, ${ }^{3}$ K. Kinoshita, ${ }^{3}$ I. C. Lai, ${ }^{3}$ P. Pomianowski, ${ }^{3}$ S. Schrenk, ${ }^{3}$ G. Bonvicini, ${ }^{4}$

D. Cinabro, ${ }^{4}$ R. Greene, ${ }^{4}$ L. P. Perera, ${ }^{4}$ B. Barish,${ }^{5}$ M. Chadha, ${ }^{5}$ S. Chan,${ }^{5}$ G. Eigen,${ }^{5}$

J. S. Miller, ${ }^{5}$ C. O'Grady, ${ }^{5}$ M. Schmidtler, ${ }^{5}$ J. Urheim, ${ }^{5}$ A. J. Weinstein, ${ }^{5}$ F. Würthwein, ${ }^{5}$

D. M. Asner ${ }^{6}$ D. W. Bliss, ${ }^{6}$ W. S. Brower,${ }^{6}$ G. Masek,${ }^{6}$ H. P. Paar,${ }^{6}$ V. Sharma,${ }^{6}$

J. Gronberg, ${ }^{7}$ R. Kutschke, ${ }^{7}$ D. J. Lange, ${ }^{7}$ S. Menary, ${ }^{7}$ R. J. Morrison, ${ }^{7}$ H. N. Nelson, ${ }^{7}$

T. K. Nelson, ${ }^{7}$ C. Qiao, ${ }^{7}$ J. D. Richman,${ }^{7}$ D. Roberts,${ }^{7}$ A. Ryd,${ }^{7}$ M. S. Witherell,${ }^{7}$

R. Balest, ${ }^{8}$ B. H. Behrens, ${ }^{8}$ K. Cho, ${ }^{8}$ W. T. Ford,${ }^{8}$ H. Park,${ }^{8}$ P. Rankin, ${ }^{8}$ J. Roy, ${ }^{8}$

J. G. Smith, ${ }^{8}$ J. P. Alexander,${ }^{9}$ C. Bebek,${ }^{9}$ B. E. Berger,${ }^{9}$ K. Berkelman, ${ }^{9}$ K. Bloom,${ }^{9}$

D. G. Cassel,${ }^{9}$ H. A. Cho, ${ }^{9}$ D. M. Coffman, ${ }^{9}$ D. S. Crowcroft,${ }^{9}$ M. Dickson, ${ }^{9}$ P. S. Drell, ${ }^{9}$

K. M. Ecklund, ${ }^{9}$ R. Ehrlich, ${ }^{9}$ R. Elia,${ }^{9}$ A. D. Foland,${ }^{9}$ P. Gaidarev,${ }^{9}$ B. Gittelman, ${ }^{9}$

S. W. Gray, ${ }^{9}$ D. L. Hartill, ${ }^{9}$ B. K. Heltsley, ${ }^{9}$ P. I. Hopman, ${ }^{9}$ J. Kandaswamy, ${ }^{9}$

N. Katayama, ${ }^{9}$ P. C. Kim,${ }^{9}$ D. L. Kreinick, ${ }^{9}$ T. Lee,${ }^{9}$ Y. Liu, ${ }^{9}$ G. S. Ludwig, ${ }^{9}$ J. Masui, ${ }^{9}$

J. Mevissen, ${ }^{9}$ N. B. Mistry, ${ }^{9}$ C. R. Ng, ${ }^{9}$ E. Nordberg, ${ }^{9}$ M. Ogg, ${ }^{9}$, J. R. Patterson, ${ }^{9}$

D. Peterson, ${ }^{9}$ D. Riley, ${ }^{9}$ A. Soffer, ${ }^{9}$ C. Ward,${ }^{9}$ M. Athanas, ${ }^{10}$ P. Avery,${ }^{10}$ C. D. Jones,${ }^{10}$

M. Lohner, ${ }^{10}$ C. Prescott,${ }^{10}$ S. Yang, ${ }^{10}$ J. Yelton, ${ }^{10}$ J. Zheng, ${ }^{10}$ G. Brandenburg,,${ }^{11}$

R. A. Briere ${ }^{11}$ Y.S. Gao, ${ }^{11}$ D. Y.-J. Kim,${ }^{11}$ R. Wilson,${ }^{11}$ H. Yamamoto,${ }^{11}$ T. E. Browder,${ }^{12}$

F. Li, ${ }^{12}$ Y. Li, ${ }^{12}$ J. L. Rodriguez, ${ }^{12}$ T. Bergfeld, ${ }^{13}$ B. I. Eisenstein, ${ }^{13}$ J. Ernst,${ }^{13}$

G. E. Gladding, ${ }^{13}$ G. D. Gollin, ${ }^{13}$ R. M. Hans ${ }^{13}$ E. Johnson, ${ }^{13}$ I. Karliner, ${ }^{13}$ M. A. Marsh, ${ }^{13}$

M. Palmer, ${ }^{13}$ M. Selen, ${ }^{13}$ J. J. Thaler, ${ }^{13}$ K. W. Edwards, ${ }^{14}$ A. Bellerive, ${ }^{15}$ R. Janicek, ${ }^{15}$

D. B. MacFarlane, ${ }^{15}$ K. W. McLean, ${ }^{15}$ P. M. Patel,${ }^{15}$ A. J. Sadoff, ${ }^{16}$ R. Ammar, ${ }^{17}$

P. Baringer ${ }^{17}$ A. Bean, ${ }^{17}$ D. Besson, ${ }^{17}$ D. Coppage,${ }^{17}$ C. Darling, ${ }^{17}$ R. Davis,${ }^{17}$

N. Hancock, ${ }^{17}$ S. Kotov ${ }^{17}$ I. Kravchenko, ${ }^{17}$ N. Kwak, ${ }^{17}$ S. Anderson, ${ }^{18}$ Y. Kubota, ${ }^{18}$

M. Lattery, ${ }^{18}$ J. J. O'Neill, ${ }^{18}$ S. Patton, ${ }^{18}$ R. Poling, ${ }^{18}$ T. Riehle, ${ }^{18}$ V. Savinov, ${ }^{18}$ A. Smith, ${ }^{18}$

M. S. Alam, ${ }^{19}$ S. B. Athar, ${ }^{19}$ Z. Ling, ${ }^{19}$ A. H. Mahmood,${ }^{19}$ H. Severini, ${ }^{19}$ S. Timm,,${ }^{19}$

F. Wappler, ${ }^{19}$ A. Anastassov ${ }^{20}$ S. Blinov, ${ }^{20}$, J J. E. Duboscq ${ }^{20}$ K. D. Fisher, ${ }^{20}$ D. Fujino, ${ }^{20, \beta}$

R. Fulton, ${ }^{20}$ K. K. Gan, ${ }^{20}$ T. Hart,${ }^{20}$ K. Honscheid,${ }^{20}$ H. Kagan, ${ }^{20}$ R. Kass,${ }^{20}$ J. Lee,${ }^{20}$

M. B. Spencer, ${ }^{20}$ M. Sung, ${ }^{20}$ A. Undrus, ${ }^{20,2}$ R. Wanke, ${ }^{20}$ A. Wolf, ${ }^{20}$ M. M. Zoeller, ${ }^{20}$

B. Nemati, ${ }^{21}$ S. J. Richichi, ${ }^{21}$ W. R. Ross,${ }^{21}$ P. Skubic, ${ }^{21}$ M. Wood,${ }^{21}$ M. Bishai, ${ }^{22}$ J. Fast, ${ }^{22}$

E. Gerndt, ${ }^{22}$ J. W. Hinson, ${ }^{22}$ N. Menon, ${ }^{22}$ D. H. Miller, ${ }^{22}$ E. I. Shibata, ${ }^{22}$ I. P. J. Shipsey ${ }^{22}$

M. Yurko, ${ }^{22}$ L. Gibbons, ${ }^{23}$ S. D. Johnson, ${ }^{23}$ Y. Kwon, ${ }^{23}$ S. Roberts,${ }^{23}$ E. H. Thorndike, ${ }^{23}$

C. P. Jessop, ${ }^{24}$ K. Lingel, ${ }^{24}$ H. Marsiske, ${ }^{24}$ M. L. Perl,${ }^{24}$ S. F. Schaffner, ${ }^{24}$ D. Ugolini, ${ }^{24}$

R. Wang, ${ }^{24}$ X. Zhou, ${ }^{24}$ T. E. Coan, ${ }^{25}$ V. Fadeyev, ${ }^{25}$ I. Korolkov, ${ }^{25}$ Y. Maravin, ${ }^{25}$

I. Narsky, ${ }^{25}$ V. Shelkov, ${ }^{25}$ J. Staeck, ${ }^{25}$ R. Stroynowski, ${ }^{25}$ I. Volobouev, ${ }^{25}$ and J. Ye ${ }^{25}$

${ }^{1}$ Syracuse University, Syracuse, New York 13244

\footnotetext{
${ }^{1}$ Permanent address: University of Texas, Austin TX 78712

${ }^{2}$ Permanent address: BINP, RU-630090 Novosibirsk, Russia.

${ }^{3}$ Permanent address: Lawrence Livermore National Laboratory, Livermore, CA 94551.
} 
${ }^{2}$ Vanderbilt University, Nashville, Tennessee 37235

${ }^{3}$ Virginia Polytechnic Institute and State University, Blacksburg, Virginia 24061

${ }^{4}$ Wayne State University, Detroit, Michigan 48202

${ }^{5}$ California Institute of Technology, Pasadena, California 91125

${ }^{6}$ University of California, San Diego, La Jolla, California 92093

${ }^{7}$ University of California, Santa Barbara, California 93106

${ }^{8}$ University of Colorado, Boulder, Colorado 80309-0390

${ }^{9}$ Cornell University, Ithaca, New York 14853

${ }^{10}$ University of Florida, Gainesville, Florida 32611

${ }^{11}$ Harvard University, Cambridge, Massachusetts 02138

${ }^{12}$ University of Hawaii at Manoa, Honolulu, Hawaii 96822

${ }^{13}$ University of Illinois, Champaign-Urbana, Illinois 61801

${ }^{14}$ Carleton University, Ottawa, Ontario, Canada K1S 5B6 and the Institute of Particle Physics, Canada

${ }^{15}$ McGill University, Montréal, Québec, Canada H3A 2T8 and the Institute of Particle Physics, Canada

${ }^{16}$ Ithaca College, Ithaca, New York 14850

${ }^{17}$ University of Kansas, Lawrence, Kansas 66045

${ }^{18}$ University of Minnesota, Minneapolis, Minnesota 55455

${ }^{19}$ State University of New York at Albany, Albany, New York 12222

${ }^{20}$ Ohio State University, Columbus, Ohio 43210

${ }^{21}$ University of Oklahoma, Norman, Oklahoma 73019

${ }^{22}$ Purdue University, West Lafayette, Indiana 47907

${ }^{23}$ University of Rochester, Rochester, New York 14627

${ }^{24}$ Stanford Linear Accelerator Center, Stanford University, Stanford, California 94309

${ }^{25}$ Southern Methodist University, Dallas, Texas 75275 
It is often argued that the individual semileptonic branching fractions, $b_{+}$and $b_{0}$, of $B^{+}$and $B^{0}$ should be similar, that a heavy quark meson should decay weakly without regard to the flavor of the light spectator quark. Strictly speaking, however, weak hadronic decays are not isospin invariant; in the case of charm, the asymmetry of hadronic widths is known to be large. For $b$-hadrons, the resulting deviation from unity of the lifetime ratio $\tau\left(B^{+}\right) / \tau\left(B^{0}\right)$ is predicted to be of order $10 \%$ [1]. There has also been some speculation that differences will be manifested in the semileptonic decay spectra [2].

The method of partial reconstruction of $B^{0} \rightarrow D^{*-} \ell^{+} \nu$, where the decay $D^{*-} \rightarrow \bar{D}^{0} \pi^{-}$is identified using only the $\pi^{-}$, has been used previously by CLEO II, to measure both the mixing parameter $\chi_{d}$ [3, 4 and semileptonic branching fraction $b_{0}$, 4, 50 of $B^{0}$. We present here an analysis to measure the leptonic spectrum of an event sample highly enriched in $B^{0}$ mesons through the application of partial reconstruction as a tag. This is, with minor corrections, a measurement of the inclusive leptonic spectrum from semileptonic $B^{0}$ decays. It may be compared with the inclusive spectrum in $\Upsilon(4 \mathrm{~S})$ events, which contains a roughly equal mix of $B^{0}$ and $B^{+}$. By comparing the rates in untagged and tagged events, it is possible to extract not only a value for $b_{0}$, but also for $b_{+}$and $\beta \equiv b_{+} / b_{0}$. Assuming that the exclusive and inclusive semileptonic partial widths are equal for $B^{0}$ and $B^{+}, \beta$ is then equal to the ratio of charged to neutral $B$ lifetimes $\tau_{+} / \tau_{0}$.

The data, collected with the CLEO II detector [6] at the Cornell Electron Storage Ring (CESR), consist of integrated luminosities $2.38 \mathrm{fb}^{-1}$ on the $\Upsilon(4 S)$ resonance and $1.13 \mathrm{fb}^{-1}$ at a center-ofmass energy which is lower by $60 \mathrm{MeV}$ (continuum). All events considered here must have at least 5 well-fitted charged tracks, a measured energy at least 0.15 times the CM energy and an event vertex consistent with the known interaction point.

$B^{0}$ decays are identified in the mode $B^{0} \rightarrow D^{*-} \ell^{+} \nu\left(D^{*-} \rightarrow \bar{D}^{0} \pi^{-}\right)$(inclusion of the charge conjugate decay is implied throughout this paper), where only the $\ell^{+}$and $\pi^{-}$are detected. A large sample of $B^{0}$ is obtained by exploiting the extremely low energy of the $D^{*-}$ decay. The method is described fully in Ref. [4. In brief, the momentum of the $\pi^{-}$is scaled to obtain an approximate four-momentum $\left(\widetilde{E}_{D^{*}}, \widetilde{\mathbf{p}}_{D^{*}}\right)$ for the $D^{*-}$ :

$$
\begin{gathered}
E_{D^{*}} \simeq \frac{E_{\pi}}{E_{\pi}^{C M}} M_{D^{*}} \equiv \widetilde{E}_{D^{*}} \\
\mathbf{p}_{D^{*}} \simeq \hat{\mathbf{p}}_{\pi} \times \sqrt{\widetilde{E}_{D^{*}}^{2}-M_{D^{*}}^{2}} \equiv \widetilde{\mathbf{p}}_{D^{*}}
\end{gathered}
$$

where $E_{\pi}$ is the $\pi^{-}$energy, $E_{\pi}^{C M}=145 \mathrm{MeV}$ is the energy of the $\pi^{-}$in the $D^{*-}$ rest frame, and $M_{D^{*}}$ is the mass of the $D^{*-}$. Using the approximation $\mathbf{p}_{\mathbf{B}} \simeq 0$ allows us to calculate a squared missing mass,

$$
\widetilde{M}_{\nu}^{2} \equiv\left(E_{\text {beam }}-\widetilde{E}_{D^{*}}-E_{\ell}\right)^{2}-\left(\widetilde{\mathbf{p}}_{D^{*}}+\mathbf{p}_{\ell}\right)^{2}
$$

which approximates the squared mass of the neutrino. Nearly all $\ell^{+} \pi^{-}$candidates stemming from decays $B \rightarrow D^{*-}(\pi) \ell^{+} \nu\left(D^{*-} \rightarrow \bar{D}^{0} \pi^{-}\right)$, where the pion is the daughter of $D^{*-}$, fall in the signal region, $\widetilde{M}_{\nu}^{2}>-2.0 \mathrm{GeV}^{2}$. Such candidates constitute our tag while all other $\ell^{+} \pi^{-}$combinations constitute the background.

The number of tags is counted in the entire data set and in subsets which contain additional leptons. To avoid confusion, the lepton used in the tag candidate will be referred to hereafter as the "tag lepton", while the additional lepton from the remainder of the event will be called the "second lepton". We allow more than one "second lepton" to exist in an event. By sorting event subsets in 
$100 \mathrm{MeV} / \mathrm{c}$ increments of the momentum of the second electron or muon, we measure their spectral distributions, which may then be compared to the corresponding inclusive lepton spectra [7].

Each tag lepton track must have a momentum in the range 1.4-2.4 GeV/c and be consistent with originating at the interaction point. Electrons are identified primarily by the ratio of calorimetric energy to momentum and specific ionization $(d E / d x)$ in the drift chamber. Muons must penetrate at least five nuclear absorption lengths.

The pion in a tag candidate must be consistent with originating at the interaction point, have charge opposite to that of the lepton, and have momentum less than $0.190 \mathrm{GeV} / \mathrm{c}$. If $d E / d x$ information is available, it must be consistent with the pion hypothesis.

Second electrons (muons) are required to have momentum between $0.6 \mathrm{GeV} / \mathrm{c}(1.5 \mathrm{GeV} / \mathrm{c}$ ) and $2.5 \mathrm{GeV} /$ c. Since $B^{0}$ mixes with $\bar{B}^{0}$, we accept second leptons of either the same or opposite sign relative to the tag lepton. The tracking criteria are identical to those for the tag lepton except that the track polar angles are more restricted, $\left|\cos \theta_{\ell}\right|<0.61$ for muons and $\left|\cos \theta_{\ell}\right|<0.707$ for electrons, where $\theta_{\ell}$ is the track polar angle. By matching the tracking and identification criteria to those used in the determination of the inclusive spectra [7], we minimize systematic uncertainties in our comparison of the two. To eliminate multiply reconstructed tracks, a second lepton candidate is rejected if the angle between its momentum and that of the tag lepton is small, $\cos \theta_{\ell \ell}>0.99$.

To obtain the net number of tags in each event subset, we select candidates in the signal region $\left(\widetilde{M}_{\nu}^{2}>-2.0 \mathrm{GeV}^{2}\right)$ and subtract backgrounds. The nonresonant contribution is estimated using the continuum dataset, corrected for luminosity and energy differences. Where a second lepton is selected, we account for cases where this second lepton is a misidentified hadron (fake) by repeating the above process selecting, in place of second leptons, tracks not identified as leptons but passing all other second lepton requirements. The result is scaled by the fake rate per track at the appropriate momentum [7]. After correction for fakes, the remaining background, from candidates which are random combinations of leptons and pions in $B \bar{B}$ events, is estimated via Monte Carlo. Its normalization is obtained from the sideband region, $-20<\widetilde{M}_{\nu}^{2}<-4 \mathrm{GeV}^{2}$. The set of events used for this estimate is approximately five times the size of the data set. The bins with second lepton momentum in the range 2.1-2.5 GeV/c are combined for the Monte Carlo sample to improve the statistical significance of the background estimate. The statistical error of the Monte Carlo sample is included with that of the data and contributes approximately $10 \%$ to its magnitude. Tags that are counted more than once within the signal region because the soft pion curls and is multiply reconstructed are found in Monte Carlo to occur for about $4 \%$ of the signal. This overcounting cancels in the ratio of tagging rates which is taken for the final result. The resulting overestimate of the statistics is accounted for by increasing the statistical errors by $2 \%$.

The evaluation of background in the signal region depends on an accurate shape for the simulated background distribution in $\widetilde{M}_{\nu}^{2}$. To quantify the uncertainty on this, we repeat the analysis using "wrong-sign" candidates, where the tag lepton and pion carry the same charge and no net signal is expected. We obtain a net "signal" of $-277 \pm 211$ events from the entire event set. This is consistent with zero, and we take as the systematic uncertainty the absolute value of the mean plus one standard deviation, which corresponds to $3.0 \%$ of raw candidates in the wrong sign signal region. We then take the uncertainty in the right sign background of each event subset to be the same fraction. The resulting systematic errors for tags with a second lepton are found to be $1.4 \%-2.1 \%$ of the net signals. We assign an overall error of $2.1 \%$ to the second lepton spectrum.

The net number of tags in the entire data set, $N_{T}$, is $22,880 \pm 283 \pm 491$. Figure 目 shows $\widetilde{M}_{\nu}^{2}$ distributions with estimated backgrounds for the full data set and for subsets corresponding to 
several lepton momentum bins. The net number of tags with second leptons above $1.5 \mathrm{GeV} / \mathrm{c}$ is $1268 \pm 54$ (statistical error only).

The net number of tags in each bin of second lepton momentum is now corrected for the lepton identification efficiency. We use the combined efficiency for geometric acceptance, track reconstruction, and identification as a function of momentum which is found in Ref. [7].

We now estimate and subtract the contribution from $\psi$ decay. The spectrum of electrons or muons from $\psi$ decay which accompany selected tags is found using Monte Carlo $B \bar{B}$ events. It is then scaled to the number of $\psi$ 's in data. The scaling factor is determined through reconstruction of decays $\psi \rightarrow \mu^{+} \mu^{-}$in the full event set and in Monte Carlo.

The remaining second leptons can be considered as either primary, produced directly in semileptonic $B$ decay, or secondary, from products of $B$ decay. To assess the contribution from secondary decay, the tagged electron and muon spectra are now fitted simultaneously to sums of primary and secondary shapes, as was done for the inclusive spectra [7]. The primary shape is derived using the model of Altarelli et al. [8]. Secondary leptons originate from decays of charm $\left(D, D_{s}, \Lambda_{c}\right)$ and $\tau$, as well as (for electrons) $\pi^{0}$ Dalitz decays and conversions of photons in the beam pipe. Above $0.6 \mathrm{GeV} / \mathrm{c}$, the spectra from all of these are similar in shape. They are therefore approximated using the spectrum from $D$ 's. The inclusive spectrum of Ref. [7], which was explicitly corrected for all but the $D$ contribution, was fitted using the same shape. The tagged spectra, normalized to $N_{T}$, are shown with fits in Figure 2. Their overall appearance differs from that of the inclusive spectra because the secondary rate is higher relative to the primary rate; as one primary lepton is in the tag, there is at most one other primary lepton, whereas there can be two secondaries from charm.

The secondary contribution is now subtracted from both tagged and inclusive spectra. We then apply two corrections to the tagged spectrum. The first, arising from the efficiency of requirements on $\cos \theta_{\ell \ell}$ and on track multiplicity, has a net value of $+1.1 \pm 1.1 \%$ [ [4]. The second arises because the tagging efficiency has a slight dependence on the decay charge multiplicity of the other $B$ in an event, such that if the other decay is semileptonic, its lower average multiplicity results in a higher tag efficiency. The correction was calculated for each momentum bin of the second lepton using a Monte Carlo simulation and found to be independent of second lepton momentum, with an average value of $(-2.9 \pm 1.3) \%$. The net correction to the tagged rate from the two sources is $(-1.8 \pm 1.7) \%$.

The tagged spectra of electrons and muons are then divided by the corresponding inclusive spectra, bin-by-bin. If the primary spectra have the same shape, the resulting distribution should be flat, with a value equal to $R \equiv b_{t a g} / b_{i n c l}$, where $b_{t a g}$ and $b_{i n c l}$ are the semileptonic branching fractions measured in the tagged and total events samples, respectively. The distributions appear indeed to be flat. They are thus fitted to a constant function, where we now restrict the momentum range to $1.5-2.4 \mathrm{GeV} / \mathrm{c}$ in order to limit uncertainties associated with the secondary spectrum (Figure 3). The fits yield $R=1.013 \pm 0.061(1.036 \pm 0.071)$ with $\chi^{2} / D O F=7.5 / 8(3.1 / 8)$ for electrons (muons). The weighted average is

$$
R=1.023 \pm 0.046 \text {. }
$$

We also obtain $R$ by taking the ratio of the primary contributions to the spectra from the fits described above, and find excellent agreement.

Because the procedures for obtaining tagged and inclusive spectra have been made nearly identical, many of the associated systematic errors are highly correlated and cancel approximately in 
the ratio $R$. Among these are errors from lepton tracking and identification efficiencies, subtraction of the $\psi$ contribution, fake rates, and spectral shapes for fitting to find the secondary contribution. To estimate the effects of variations in the $\psi$ contribution and fake efficiencies, we repeat our measurement, adjusting each contribution in turn to the limits of the estimated uncertainty. To assess the uncertainty due to shapes used in fitting to obtain the secondary contribution, fitting was repeated with varying primary and secondary shapes. For the primary shape we include the model of Isgur et al. [9] where the fractional contribution of $B \rightarrow D^{* *} l \nu$ to semileptonic decays is fixed for each fit in the range $(23 \pm 1) \%$ []] (ISGW $\left.{ }^{* *}\right)$. For the secondary spectrum we use the method and shapes used in Ref. [3]. These correlated sources and effects are summarized in Table [.

Uncertainties which apply to the tagged but not to the inclusive measurement include effects due to event selection, angular cuts, and tagging efficiency differences discussed above, which contribute a total of $1.7 \%$. The uncertainty from the background estimate for the tag cancels approximately in dividing by $N_{T}$, and we assign an overall error of $1 \%$ to the magnitude of the tagged spectrum from this source. In addition, we approximate the spectra of all secondary leptons in the tagged spectra by those from $D$ decay only. To estimate the uncertainty from this and from the fact that there may be small differences in shape between tagged and inclusive samples due to tag selection itself, we repeat the analysis after subtracting an estimated contribution of secondaries from sources other than $D$ decay. This causes a shift of $1.7 \%$, which is taken to be the error. The net systematic error on the value of $R$ is $3.5 \%$.

$R$ may be represented by an equation in terms of $b_{0}, b_{+}, \beta \equiv b_{+} / b_{0}$, and several other parameters, which can be solved for $b_{0}, b_{+}$, and $\beta$. The average semileptonic branching fractions of $B$ 's in the tagged and inclusive event samples, $b_{t a g}$ and $b_{i n c l}$, may be expressed as

$$
\begin{aligned}
b_{\text {incl }} & =f_{00} b_{0}+f_{+-} b_{+}=f_{00} b_{0}(1+\alpha) \\
b_{\text {tag }} & =f_{0, \text { tag }} b_{0}+f_{+, \text {tag }} b_{+}=b_{0}\left(1+f_{+, \text {tag }} \delta \beta\right),
\end{aligned}
$$

where $f_{00}$ and $f_{+-}$are the fractions of $B^{0}$ and $B^{+}$events from the $\Upsilon(4 \mathrm{~S}), f_{0, t a g}$ and $f_{+, \operatorname{tag}}$ are the corresponding fractions among tags, $\alpha \equiv f_{+-} b_{+} / f_{00} b_{0}$, and $\delta \beta \equiv \beta-1$.

From (2) and the definition of $\beta$ we obtain

$$
\begin{aligned}
b_{0} & =\frac{b_{\text {tag }}}{1+f_{+, \text {tag }} \delta \beta}=\frac{R b_{\text {incl }}}{1+f_{+, \text {tag }} \delta \beta} \\
b_{+} & =\beta b_{0}=\frac{R b_{\text {incl }}(1+\delta \beta)}{1+f_{+, \text {tag }} \delta \beta}
\end{aligned}
$$

To obtain $\delta \beta$, we take the ratio of (2) and (11)

$$
\begin{aligned}
R & \equiv \frac{b_{\text {tag }}}{b_{\text {incl }}}=\frac{b_{0}\left(1+f_{+, t a g} \delta \beta\right)}{f_{00} b_{0}(1+\alpha)}, \\
\Rightarrow f_{00} & =\frac{1+f_{+, t a g} \delta \beta}{R(1+\alpha)} .
\end{aligned}
$$

From the definitions of $\alpha$ and $\delta \beta$,

$$
f_{00}=\frac{1+\delta \beta}{\alpha+1+\delta \beta}
$$

Equating (6) and (7), 


$$
(1+\delta R)(1+\alpha)(1+\delta \beta)=\left(1+f_{+, t a g} \delta \beta\right)(\alpha+1+\delta \beta)
$$

$f_{+, t a g}, \delta \beta$, and $\delta R \equiv R-1$ are expected to be "small", $<0.1$, so we discard the highest order term, $f_{+, \text {tag }} \delta \beta^{2}$, to arrive at a linear equation in $\delta \beta$ which is solved to obtain

$$
\delta \beta=\frac{\delta R(1+\alpha)}{(1+\alpha)\left(f_{+, t a g}-\delta R\right)-\alpha} .
$$

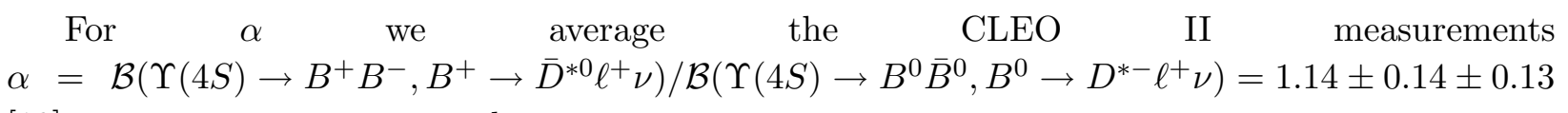
10 and $\alpha=$ $\mathcal{B}\left(\Upsilon(4 S) \rightarrow B^{+} B^{-}, B^{+} \rightarrow \psi K^{(*)+}\right) / \mathcal{B}\left(\Upsilon(4 S) \rightarrow B^{0} \bar{B}^{0}, B^{0} \rightarrow \psi K^{(*) 0}\right)=1.15 \pm 0.17 \pm 0.06$ [1], to get $\alpha=1.15 \pm 0.13$.

The fraction $f_{+, \text {tag }}$ is nonzero because some charged $B$ 's are present among our tags from decays of the type $B \rightarrow D^{*-} \pi \ell^{+} \nu$ (where the $D^{*+} \pi$ may or may not form a resonance). The fraction $f^{* *}$ of such decays in the tag sample may be determined by adding the reconstruction of the $\bar{D}^{0}$ decay, so that a measured $D^{*-}$ momentum is used in the calculation of missing mass squared. In this case the resolution is sufficient to achieve some degree of separation between the decays without and with an additional pion, so that the relative rates may be determined. In terms of our analysis the only directly applicable measurement has been performed at CLEO I, with the same event and tag lepton momentum requirements which found $f^{* *}=0.14 \pm 0.08$, the value of which can be derived from measurements cited in [12]. Because $f^{* *}$ is a ratio, it depends mainly on kinematic criteria and should not differ significantly in CLEO II. We use a measurement from ALEPH, $\mathcal{B}\left(B \rightarrow D^{*-} \pi^{+} \ell^{+} \nu X\right)=(1.25 \pm 0.16 \pm 0.12) \%$ [13], and Monte Carlo simulations to obtain an independent value, $f^{* *}=0.10 \pm 0.02$, which is in agreement with CLEO I but may have a large systematic error due to insufficiency of our current knowledge about the different modes which contribute to $B \rightarrow D^{*-} \pi^{+} \ell^{+} \nu X$. We use the CLEO I value because it is more directly obtained, and our evaluations of $b_{0}, b_{+}$, and $f_{00}$ are insensitive to it. The relative contributions to $f^{* *}$ from charged and neutral $B$ 's is determined using the approximate conservation of isospin in the fragmentation of the hadronic part of the final state; if $b_{0}$ and $b_{+}$are equal and the charged and neutral $B$ 's decay to $\bar{D}^{*} X \ell^{+} \nu$ at the same rates, the rate to states where $X$ is a charged pion is expected to be double that of the rate where it is a neutral pion. In other words, $B^{+} \rightarrow D^{*-} \pi^{+} \ell^{+} \nu$ contributes to $f^{* *}$ at twice the rate of $B^{0} \rightarrow D^{*-} \pi^{0} \ell^{+} \nu$, weighted by the respective production rates and semileptonic branching fractions [3],

$$
f_{+, t a g}=f^{* *} \frac{2 \alpha}{1+2 \alpha},
$$

from which we get $f_{+, t a g}=(9.7 \pm 5.5) \%$.

Using $R=1.023 \pm 0.046 \pm 0.036, f^{* *}=0.14 \pm 0.08$, and $\alpha=1.15 \pm 0.13$, we obtain

$$
\begin{aligned}
\beta & =0.950_{-0.080-0.068}^{+0.117+0.091}, \\
f_{+-} / f_{00} & =1.21 \pm 0.12 \pm 0.17,
\end{aligned}
$$

where in each case the first error is statistical and the second systematic. The latter number may be expressed $f_{00}=0.452 \pm 0.028 \pm 0.038$. The values of $b_{0}$ and $b_{+}$may be obtained with the addition of $b_{\text {incl }}$. The most recent published measurement of an inclusive semileptonic branching fraction at the $\Upsilon(4 \mathrm{~S})$ was made using a lepton tagging method which gives a value that is not strictly equal to 
$b_{\text {incl }}$ [14], as the tagged event sample may not have the same composition as generic $\Upsilon(4 \mathrm{~S})$ events. However, given known limits on the input parameters, it is expected to differ from $b_{\text {incl }}$ by less than one part in $10^{2}$, so we use the result, $b_{\text {incl }} \approx(10.49 \pm 0.46) \%$, to get

$$
\begin{aligned}
b_{0} & =(10.78 \pm 0.60 \pm 0.69) \%, \\
b_{+} & =(10.25 \pm 0.57 \pm 0.65) \% .
\end{aligned}
$$

As the determinations of $b_{0}, b_{+}, \beta$, and $f_{+-} / f_{00}$ involve correlated inputs, the errors are obtained numerically. All of the statistical uncertainties are defined by the uncertainty in $R$. The dominant contributors to the systematic uncertainties are $R$ for $\beta$, both $R$ and $b_{i n c l}$ for $b_{0}$ and $b_{+}$, and $\alpha$ for $f_{00}$.

Our results are consistent with our previous ones [5], $\beta=0.93 \pm 0.18 \pm 0.12, b_{0}=(10.9 \pm 0.7 \pm$ $1.1) \%$, and $b_{+}=(10.1 \pm 1.8 \pm 1.5) \%$. Our new result for $b_{0}$ is somewhat correlated with the previous one, as the data sets overlap partially and parts of the method are similar. The results for $b_{+}$are uncorrelated. $f_{+-} / f_{00}$ may also be obtained using the CLEO measurement of $\alpha$ with a lifetime ratio based on measurements at LEP and CDF, as in [10]. The value reported here was obtained using CLEO data exclusively.

To summarize, the electronic and muonic spectra have been measured in a sample of events enriched in neutral $B$ 's using partial reconstruction tags. By comparing these spectra to the inclusive spectra in $\Upsilon(4 \mathrm{~S})$ events, we probe differences between $B^{0}$ and $B^{+}$in rates and spectral shapes of primary semileptonic decay. We observe no evidence for differences in the spectra between 1.5 and $2.4 \mathrm{GeV} / \mathrm{c}$. Assuming the shapes are the same, we obtain $b_{0}, b_{+}, \beta$, and $f_{+-} / f_{00}$. Our new value of $f_{+-} / f_{00}$ is the first that is independent of $B$ meson lifetime measurements.

\section{ACKNOWLEDGEMENTS}

We gratefully acknowledge the effort of the CESR staff in providing us with excellent luminosity and running conditions. J.P.A., J.R.P., and I.P.J.S. thank the NYI program of the NSF, M.S. thanks the PFF program of the NSF, G.E. thanks the Heisenberg Foundation, K.K.G., M.S., H.N.N., T.S., and H.Y. thank the OJI program of DOE, J.R.P., K.H., M.S. and V.S. thank the A.P. Sloan Foundation, and A.W. and R.W. thank the Alexander von Humboldt Stiftung for support. M.S. is supported as a Cottrell Scholar of Research Corporation. This work was supported by the National Science Foundation, the U.S. Department of Energy, and the Natural Sciences and Engineering Research Council of Canada. 


\section{REFERENCES}

[1] I. I. Bigi et al., in B Decays, 2nd edition, S. Stone, ed. (World Scientific 1994).

[2] I.I. Bigi and N.G. Uraltsev, Nucl. Phys. B 423, 33 (1994).

[3] J. Bartelt et al. (CLEO), Phys. Rev. Lett. 71, 1680 (1993).

[4] M.S. Saulnier, Ph.D. thesis, Harvard University, 1995 (unpublished).

[5] M. Athanas et al. (CLEO), Phys. Rev. Lett. 73, 3503 (1994); Phys. Rev. Lett. 74, 3090 (1995).

[6] Y. Kubota et al. (CLEO), Nucl. Instrum. Methods A 320, 66 (1992).

[7] J. Bartelt et al. (CLEO), "Inclusive Measurement of $B$ Meson Semileptonic Branching Fractions," CLEO CONF93-19; R. Wang, Ph.D. thesis, Univ. of Minnesota, 1995 (unpublished).

[8] G. Altarelli et al., Nucl. Phys. B 208, 365 (1982).

[9] N. Isgur, D. Scora, B. Grinstein, and M. Wise, Phys. Rev. D 39, 799 (1989).

[10] B. Barish et al. (CLEO), Phys. Rev. D 51, 1014 (1995).

[11] C. P. Jessop et al., preprint CLEO 96-24, submitted to Physical Review Letters.

[12] D. Bortoletto et al. (CLEO), Phys. Rev. Lett. 63, 1667 (1989).

[13] J. Richman, in Proc. XXVIII Int'l Conf. on High Energy Physics, (Warsaw, Poland 1996) in press.

[14] B. Barish et al. (CLEO), Phys. Rev. Lett. 76, 1570 (1996). 


\section{TABLES}

TABLE I. Systematic errors on $R$. Those listed in the upper section apply both to tagged and inclusive spectra, while those in the lower section apply only to the tagged spectra. For each listed source, the ratio of its magnitude to the net tagged spectrum above $1.5 \mathrm{GeV} / \mathrm{c}$ is shown in parentheses.

\begin{tabular}{ccc}
\hline \hline Source & $\begin{array}{c}\sigma_{\text {sys }} / \text { value }(\%) \\
\text { or procedure }\end{array}$ & $\delta(R) / R(\%)$ \\
\hline$\psi$ contribution $(0.011)$ & 30 & 0.3 \\
Fake electrons $(0.002)$ & 50 & 0.1 \\
Fake muons $(0.011)$ & 25 & 0.2 \\
Secondary spectrum (.044) & as in Ref. [3 & 2.3 \\
Primary spectrum (0.95) & ISGW ${ }^{* *}$ [7 & 0.3 \\
$(b \rightarrow u)(0.019)$ & 21 & - \\
\hline Event/tag selection & 1.7 & 1.7 \\
Background estimate (0.59) & 1.0 & 1.0 \\
Secondary spectrum $(0.044)$ & vary sources (see text) & 1.7 \\
\hline Net systematic error & & 3.5 \\
\hline \hline
\end{tabular}




\section{FIGURES}

3521296-001

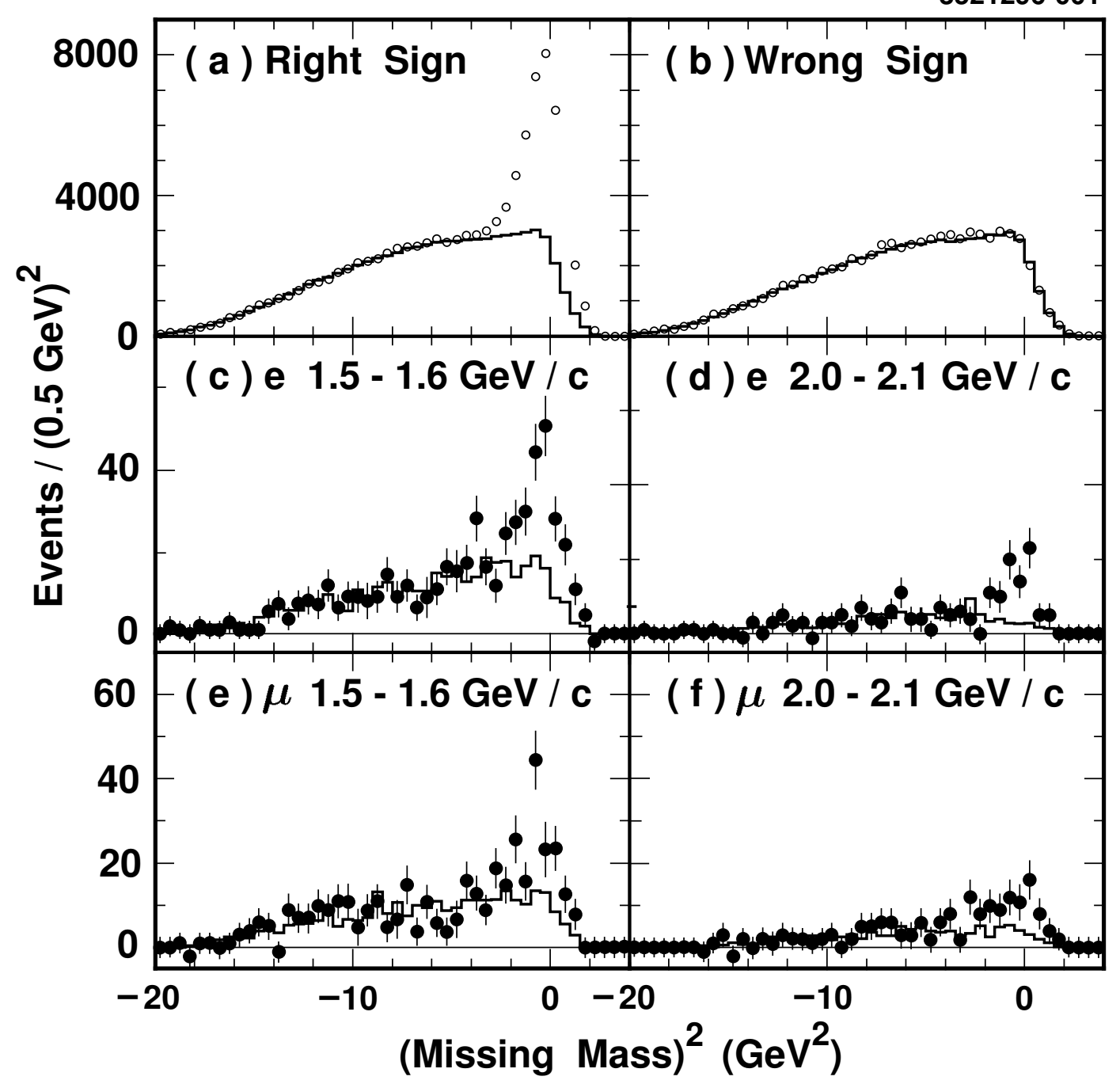

FIG. 1. Tag candidate $\widetilde{M}_{\nu}^{2}$ distributions, data (symbols) and simulated backgrounds (solid histograms). Continuum has been subtracted in all cases, and in (c)-(f), fakes have been subtracted. (a)-(b): full event set (errors are smaller than symbols), (a) opposite-sign candidates, (b) same-sign. (c)-(f): events containing a second lepton, opposite-sign candidates, selected bins as indicated. 


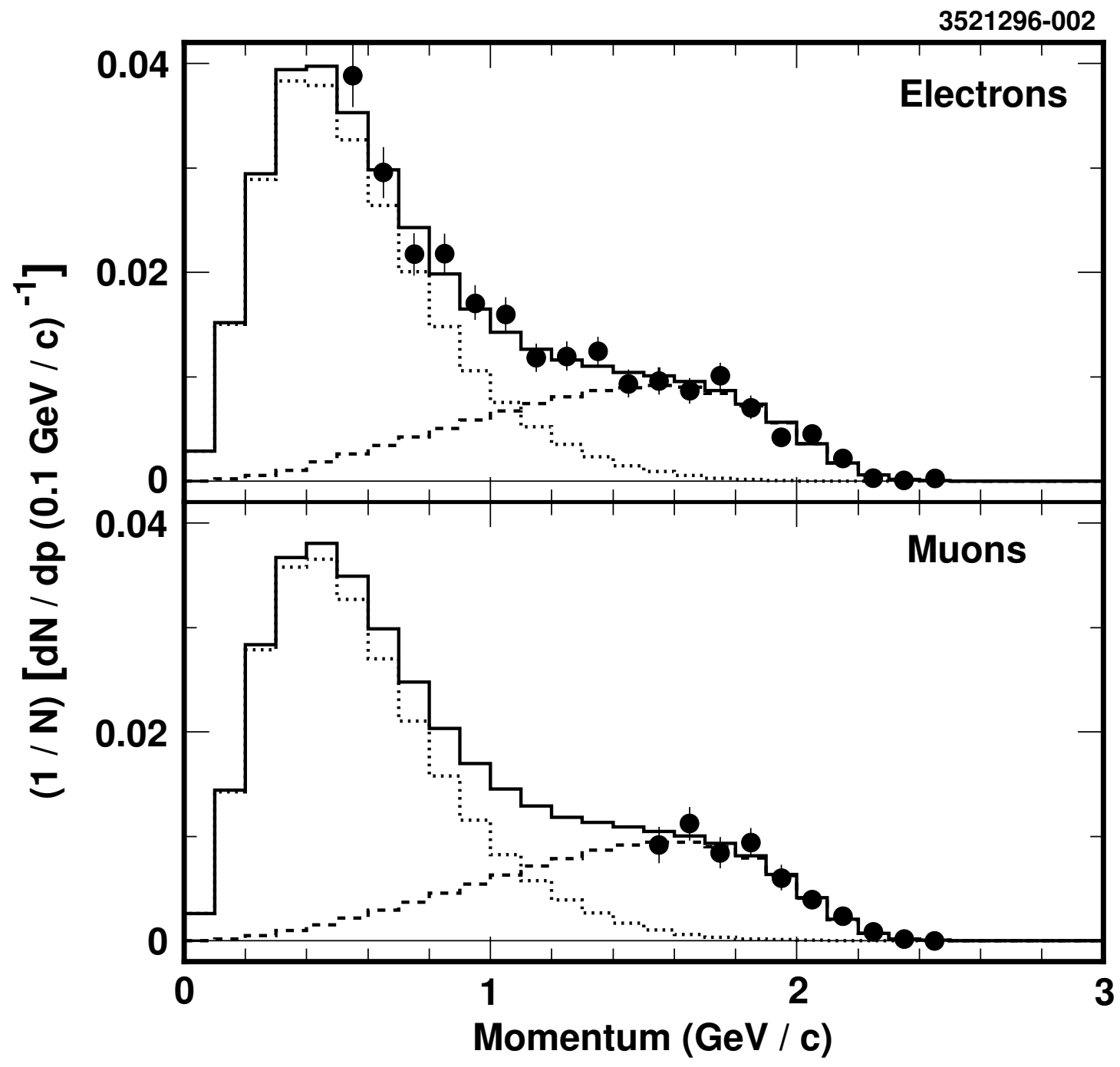

FIG. 2. Measured spectrum of second leptons, corrected for efficiency and with $\psi$ contribution subtracted, shown with fit to primary (dashed) and secondary (dotted) spectra. 


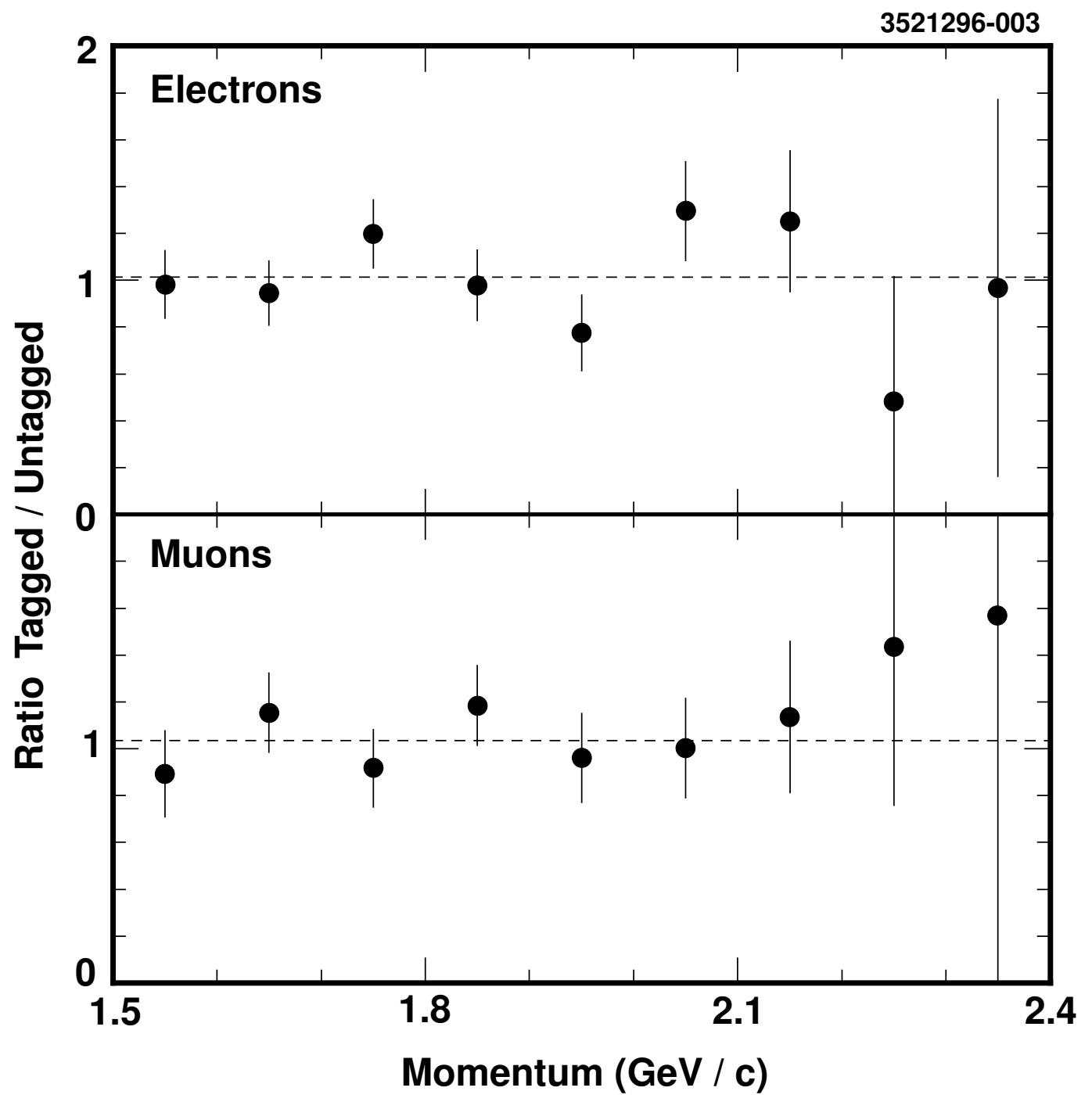

FIG. 3. Bin-by-bin ratio of tagged and inclusive spectra, with fits to a constant (dashed line) for electrons (top) and muons (bottom). 